\title{
Experimental Verification of Drill String Vibration Suppression Using an Adaptive Self-Tuning Controller
}

\author{
Fesmi Abdul Majeed and Hamad Karki \\ Mechanical Engineering Department, Petroleum Institute, Abu Dhabi, P.O. Box 2533, UAE \\ Mansour Karkoub \\ Mechanical Engineering Department, Texas A\&M, Doha, P.O. Box 23874, Qatar
}

Youssef Lotfy Abdel Magid

Electrical Engineering Department, Petroleum Institute, Abu Dhabi, P.O. Box 2533, UAE

(Received 16 November 2011, revised 20 September 2012, accepted 14 November 2012)

Drill bit whirl is a common phenomenon in rotary drilling rigs. It causes severe drill collar damage and borehole enlargement, leading to an irrevocable decrease in drilling efficiency. The majority of the research in this field concentrates on designing new drill bits or placing shock absorbers near the bottom hole assembly to minimize the damage caused by drill bit whirling. However, practically, vibrations in rotary drilling are minimized by tuning the upper rotary table speed or varying the weight on drill bit. This work explores the design and implementation of an adaptive controller to minimize vibrations of drill bits, particularly bit whirl. The developed controller achieves the vibration mitigation by varying the upper rotary speed. Moreover, the developed control law takes into account the vibrational frequencies and critical operating speeds of the drill string, thus also being capable of avoiding resonant vibrations. Experimental results are provided to prove the vibration mitigation capability of the developed controller.

\section{NOMENCLATURE}

$\begin{array}{ll}A(z) & \text { Discrete process model denominator } \\ B(z) & \text { Discrete process model numerator } \\ C(z) & \text { Discrete noise model numerator } \\ D(z) & \text { Discrete noise model denominator } \\ E & \text { Compression constant } \\ e(k) & \text { Discrete process noise vector } \\ f & \text { Controller selected frequency of operation } \\ f_{n} & \text { Natural frequency } \\ G & \text { Shear constant } \\ k & \text { Discrete time instant } \\ L & \text { Length of the drill string } \\ N & \text { Critical speed of operation } \\ n & \text { Normal frequency of operation } \\ n(t) & \text { White noise } \\ t & \text { Continuous time instant } \\ u(k) & \text { Discrete process input } \\ u(t) & \text { Process Input } \\ y(k) & \text { Discrete Process output } \\ y(t) & \text { Process output }\end{array}$

\section{INTRODUCTION}

Bit whirl is a common phenomenon arising due to high lateral vibrations in rotary drilling rigs. It causes reduction of drilling efficiency, drill pipe bending, and well borehole enlargement. Hence, lateral vibrations of the drill bit are the most destructive type of vibrations in rotary drilling. ${ }^{1-3}$

Drill bit whirl can also occur when drill bits have an imbalance in the drill bit design during manufacturing or when there is a slight bend in the drill collars due to high lateral vibrations. ${ }^{4}$ Both of these imperfections cause lateral vibrations to be predominant. ${ }^{5}$

Most of the commercially available drill bits have imbalances in the range of $2 \%$ to $10 \%$, with $2 \%$ only for a very high commercially graded bit. ${ }^{6}$ To overcome this manufacturing imbalance and reduce drill bit whirl, many new drill bit models were designed. ${ }^{6-9}$ However, these designs cannot mitigate bit whirl completely due to manufacturing imbalances. Practical solutions to minimizing and stabilizing drill string vibrations using current monitoring technology include changing the upper rotary speed or varying the weight on bit. ${ }^{10}$ This study attempts to provide a practical solution to minimizing drill bit whirl using adaptive controllers.

Adaptive controllers are a very exciting new technology, and a few researchers have recently developed and tested adaptive controllers to mitigate drill string vibrations. Fubin et al. developed an adaptive proportional-integral-derivative (PID) control strategy of the drilling rotary system to eliminate the stick-slip oscillation of the bit. ${ }^{11}$ The main objective of the adaptive PID controller is to improve system characteristics of the output-to-input and dynamic response. The results of the controller have been proven only in simulations. Jijón et al. ${ }^{12}$ designed an adaptive observer and analysed the Drilling-Oscillation Killer (D-OSKIL) control law proposed by C. Canudas-de Wit et al. ${ }^{13}$ The D-OSKIL controller uses a vertical force (weight on the bit) as an additional variable to eliminate the stick-slip effect. Jijón et al. ${ }^{12}$ tried to improve the control law with an additional adaptive observer so that the unknown states of the system could be estimated. Smoother 